\title{
Article
}

\section{An Intelligent Coaching Prototype for Elderly Care}

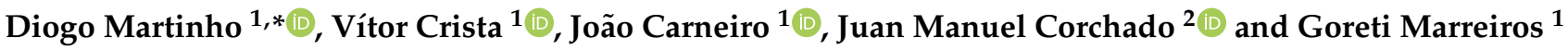 \\ 1 Research Group on Intelligent Engineering and Computing for Advanced Innovation and \\ Development (GECAD), Institute of Engineering, Polytechnic of Porto, 4200-072 Porto, Portugal; \\ 1181500@isep.ipp.pt (V.C.); jrc@isep.ipp.pt (J.C.); mgt@isep.ipp.pt (G.M.) \\ 2 BISITE Digital Innovation Hub, University of Salamanca, Edificio Multiusos, 37008 Salamanca, Spain; \\ corchado@usal.es \\ * Correspondence: diepm@isep.ipp.pt
}

check for updates

Citation: Martinho, D.; Crista, V.;

Carneiro, J.; Corchado, J.M.;

Marreiros, G. An Intelligent

Coaching Prototype for Elderly Care.

Electronics 2022, 11, 460. https://

doi.org/10.3390/electronics 11030460

Academic Editor: George

Angelos Papadopoulos

Received: 29 December 2021

Accepted: 31 January 2022

Published: 3 February 2022

Publisher's Note: MDPI stays neutral with regard to jurisdictional claims in published maps and institutional affiliations.

Copyright: (C) 2022 by the authors. Licensee MDPI, Basel, Switzerland. This article is an open access article distributed under the terms and conditions of the Creative Commons Attribution (CC BY) license (https:// creativecommons.org/licenses/by/ $4.0 /)$.

\begin{abstract}
The world ageing problem is prompting new sustainable ways to support elderly people. As such, it is important to promote personalized and intelligent ways to assure the active and healthy ageing of the population. Technological breakthroughs have led to the development of personalized healthcare systems, capable of monitoring and providing feedback on different aspects that can improve the health of the elderly person. Furthermore, defining motivational strategies to persuade the elderly person to be healthier and stay connected to such systems is also fundamental. In this work, a coaching system is presented, especially designed to support elderly people and motivate them to pursue healthier ways of living. To do this, a coaching application is developed using both a cognitive virtual assistant to directly interact with the elderly person and provide feedback on his/her current health condition, and several gamification techniques to motivate the elderly person to stay engaged with the application. Additionally, a set of simulations were performed to validate the proposed system in terms of the support and feedback provided to the user according to his progress, and through interactions with the cognitive assistant.
\end{abstract}

Keywords: coaching system; cognitive assistant; gamification

\section{Introduction}

As the world grows older, the demand for sustainable measures to assure and promote the active and healthy ageing of the population become necessary. The numbers are evident and the percentage which corresponds to the elderly part of the world's population is predicted increase in the coming years [1,2]. Therefore, the current global challenge is to understand how elderly people should be supported on a daily basis with the goal of diminishing the impact that ageing will have in terms of physical, cognitive, social and emotional deterioration [2,3]. In this scope, technology can play a fundamental role and it makes sense to take advantage of all the recent technological breakthroughs regarding the devices, methods and applications used to envision a world in which the elderly person can safely provide information about his/her current health condition and be able to receive adequate support and measures to improve it. This can be achieved by developing intelligent systems that combine information with personalized mechanisms to understand not only the preferences but also the abilities of each person, and then identify which aspects of that person's life could be improved [4-6]. Furthermore, these systems must also be aware of the fact that each person is different and will react differently even under the same scenarios [3]. Therefore, defining successful engagement strategies that can motivate the person to pursue healthier actions in his/her daily life is also essential [7-9].

Looking at the literature [3], we have learnt that many novel approaches are being introduced in this scope, which mostly rely on the use of exergames and gamified solutions with different technological features to draw user attention [10-12]. As a result, we are now seeing many works focusing on the development of mobile, console and wearable solutions. 
For example, the authors in [13] proposed a healthcare system called I-CARE using a tablet device to support elderly people with dementia, with personalized cognitive activation content and recommendations based on each person's abilities and needs. The system was designed to be used in collaboration with caregivers who guide and interact with the elderly person through each activation session. In these sessions, the elderly person and the caregivers are provided with different activation units to start conversations that represent diversified topics of interests, social backgrounds, ways of life, moral concepts, and cultural and regional socialization aspects. The authors obtained very positive results and feedback showing a broad spectrum of responses, ranging from sustained attention, and cognitive processing, to emotional reactions from the elderly person. Another work of interest was proposed in [14], in which the authors designed a mobile app with different features to support older, HIV-infected patients. The app, called +Approp included reports on clinical history (such as vaccines and medication taken), educational feedback (with motivational tools to encourage exercise and smoking cessation), reminders of medical appointments and communication tools to directly obtain support through email, messages and video calls. The authors obtained positive results regarding both usability and satisfaction from elderly people and referred to the importance of motivating elderly people to use mobile healthcare apps as a key factor to correctly manage and monitor chronic diseases. Finally, the authors in [15] referred to the importance of gamified systems to offer a wide range of new leisure activities for senior users that can improve their quality of life, and presented the work developed within the ePA-Coach project. As the main goal of this project, a digital learning environment was developed to motivate elderly people to improve their digital literacy using different gamification techniques based on the octalysis framework. This framework considers different dimensions, such as social influence, ownership, accomplishment, empowerment, among others. The authors studied which dimensions affect elderly people and defined the ePA-Coach environment with different features, such as the use of milestones, puzzles and quizzes, tasks and mentorship (among others). The authors also referred to the lack of existing gamified solutions targeted at elderly people.

Additionally, knowing the best way to interact with a certain person will also be fundamental to motivate that person to be healthier [16]. In this sense, several studies were also observed using either physical or virtual robots or assistants to directly interact with the elderly person and attempt to persuade him/her to be healthier [8,17-22]. In fact, the authors in [23] explained the importance of intelligent cognitive assistants to support attitude and behavior changes, which are crucial to protect against the deterioration of cognitive and mental health. Furthermore, other authors explain the importance of building a relationship with a virtual entity, and why this approach could be meaningful for the elderly person, promoting feelings of responsibility and even nostalgia as they to look after another person/being [24-26].

In sum, the development of intelligent mechanisms and algorithms to adjust the interaction and support provided to the elderly person, which includes serious games with scalable difficulty till robots that play games and communicate with elderly people using different emotions, is fundamental to assure interactive and personalized systems that are tailored to each user [3]. In this regard, we studied the use of processes to provide feedback and rewards to the elderly person, the possibility of increasing the cognitive/physical load as the user becomes more proficient with the system, and the adoption of methods that enhance and promote social interaction, which can result in more personalized elderly care solutions. Furthermore, adapting the support provided to the elderly person according to their interests, capabilities, and necessities, and the surrounding environment, will contribute to the improvement of health and wellbeing, capture interest and ensure positive engagement, facilitate social interaction and decrease the impact of many different medical conditions.

In this work, we present a coaching system named Coaching for Elderly (CoaFEld), specifically designed to support elderly people during their daily lives and motivate them to pursuit healthier ways of living, which will, in turn, contribute to an active and healthier ageing process. As such, we combine different ideas that were previously introduced and 
discussed [3,27-29], along with a gamified approach. By doing this, we can prove that it is possible to support the evolution of the elderly person as a player and user of the coaching system and provide him/her with different types of feedback and motivational features depending on his/her performance and progress. Our approach considers a cognitive assistant that interacts with the elderly person and reacts based on the performance of the elderly person as a player of the system. To do this, the cognitive assistant displays different emotional states to represent the person's health progress, which improve as the user improves his/her health condition. Furthermore, the proposed system offers additional gamification elements and strategies that are generally well-perceived by the elderly population and will contribute to an overall improved user experience.

We followed our previous case study to validate the proposed solution [29] and were able to observe how the coaching system would support user progress using both a reinforcement learning approach, which learns which messages are most persuasive when interacting with a certain person, and a random approach, in which the interactions are performed in a random order. We applied both approaches under different scenarios with different complexity levels and compared the results in terms of points and the emotional state of the cognitive assistant obtained in each scenario. The obtained results allowed us to confirm that the coaching system could provide adequate support and feedback to the user, depending on his/her current health condition and progress as a user of the coaching system.

The proposed work is presented as follows: in the next section, our coaching system is presented and discussed in terms of the defined architecture and its main features. Then, a case study is presented to validate the system, and the obtained results are explained and discussed. Finally, the main conclusions and the work to be done hereafter are discussed in the last section of this work.

\section{Proposed Model}

CoaFEld was developed following a microservice-oriented architecture (Figure 1). This system is composed of three main components: a user web application, an API gateway, and a set of microservices. These microservices communicate and store specific user information regarding user progress while using the application (using different gamification components), health status (derived from the performance of established coaching plans) and user interactions with the feedback that is provided to the user (personalized message interactions based on the user preferences). This information is then consumed and provided to the web application. Each of the three main components are described in more detail.

\subsection{Coaching Application}

As explained in the previous section of this work, an important decision that was taken while designing the application of the proposed system was to "gamify" the solution to not only enhance the overall quality of the solution but improve the user experience, with the goal of motivating the user to stay connected to the application. A comprehensive study was carried out on the use of gamification techniques applied to elderly care [3] to understand which kind of techniques and elements are more frequently applied in this context, and which have been more successful. We concluded with a consideration of different features that will translate the current user progress within the application, such as the use of experience/level and the use of points as the system's currency to unlock new content. All these features have already been integrated into our system, as can be seen in Figure 2. 


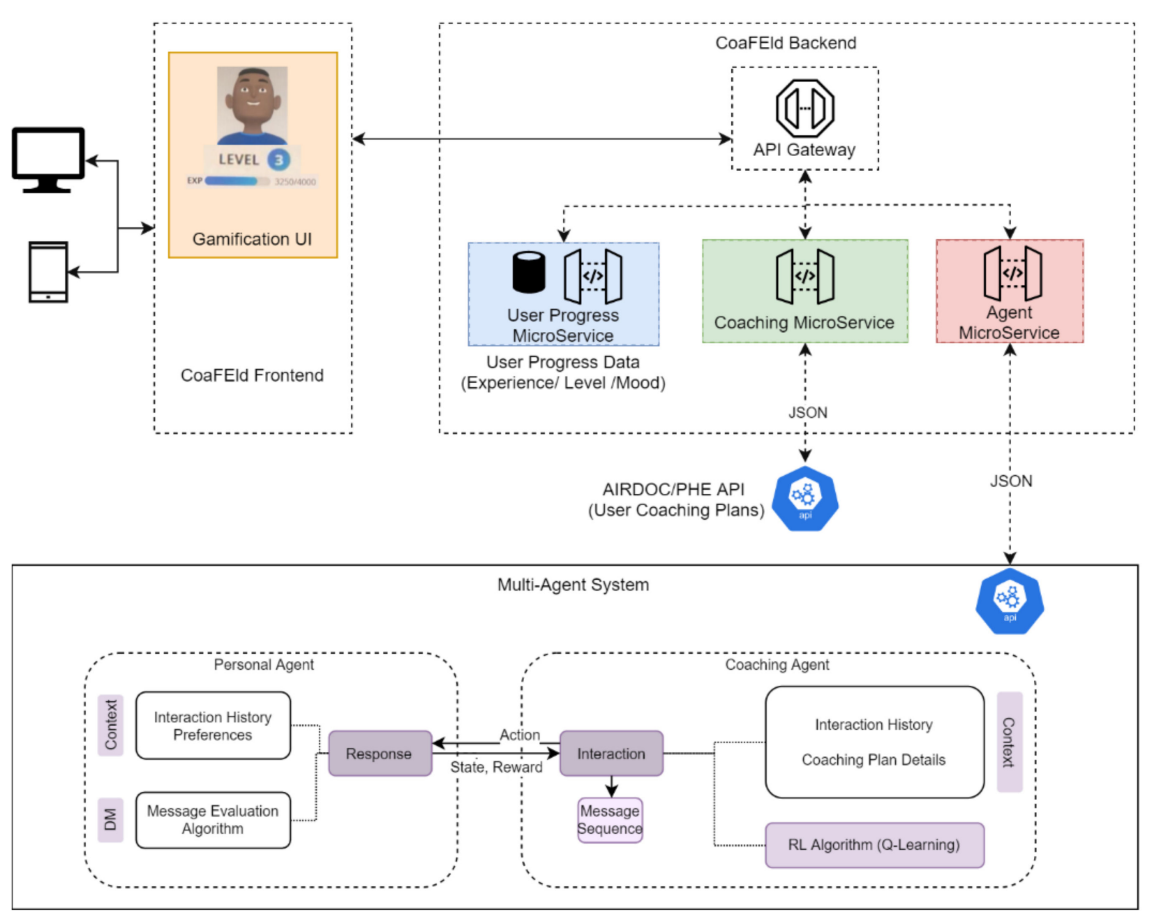

Figure 1. The proposed coaching system architecture.

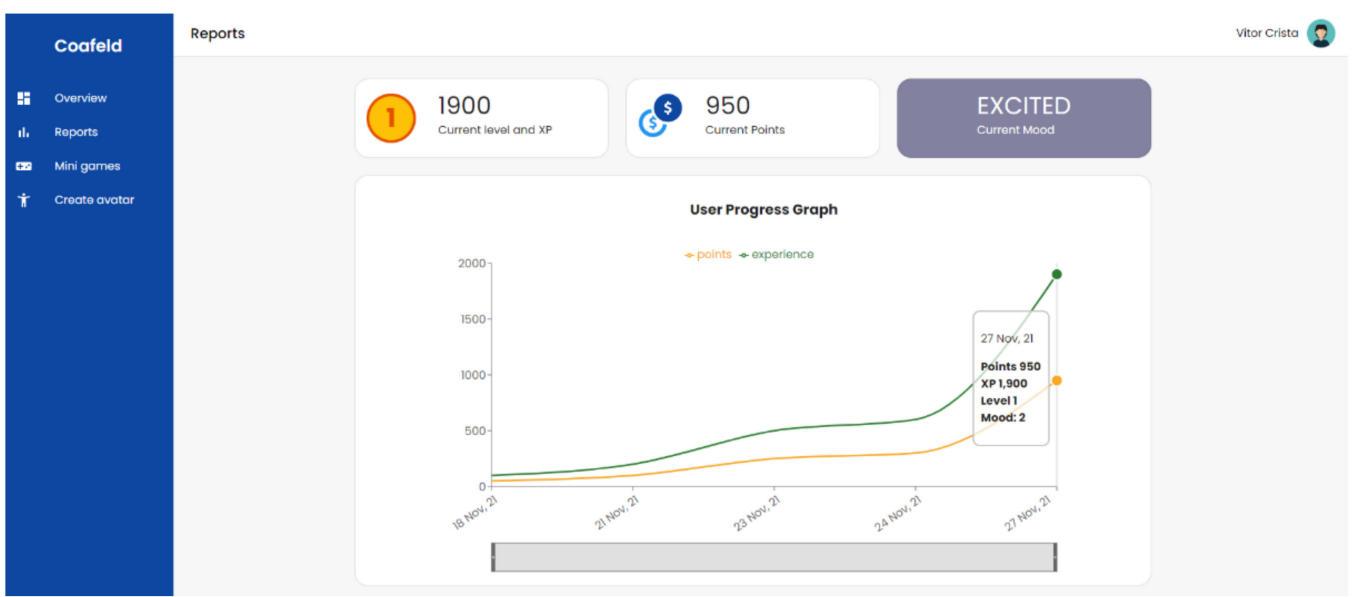

Figure 2. User progress interface. The user can access his/her current game experience in terms of points and experience acquired and the corresponding level. All points earnt can be used to unlock additional content inside the application.

The developed application also presents a cognitive virtual assistant that holds an emotional state corresponding to the user progress, which will improve as the user improves his/her health condition and decrease as the user worsens his/her health condition (see Figure 3). To measure the current emotional state of the cognitive assistant, we evaluate all coaching plans assigned to that user in terms of the number of goals that were successfully achieved by that user and the total number of goals that needed to be achieved during that coaching plan's execution. The details of these plans are described in more detail in our previous work, in [27]; however, the general idea is to establish different health goals for parameters that are monitored, which must be over/under/equal to a certain range or value. 


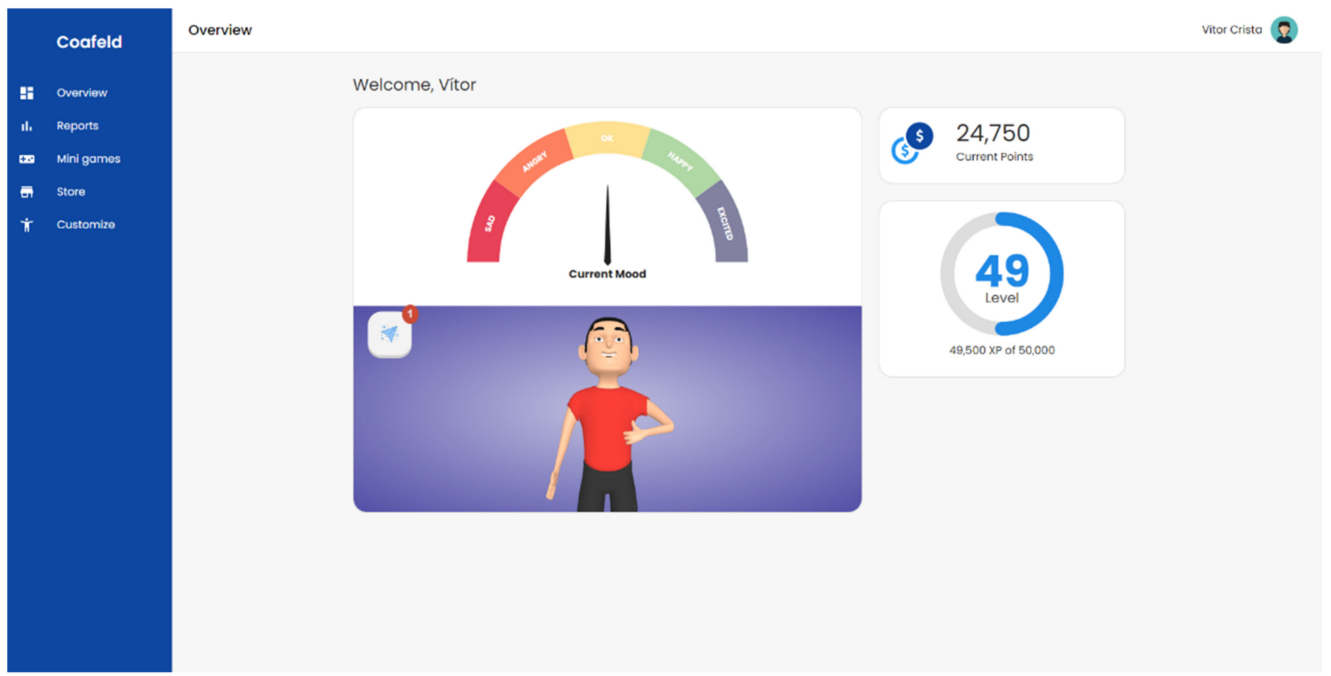

Figure 3. Main page interface. The user can see his/her current progress as well as interact with the cognitive assistant and receive feedback from the system (messages to congratulate/encourage healthier behaviors).

We consider five main emotional states to represent user progress (see Figure 4) and we applied the following formula to measure the current emotional state of the cognitive assistant:

$$
\text { emotionalState }=\frac{n A G}{t G} \times 5
$$

where:

- $n A G$ - corresponds to the number of goals achieved in the coaching plan currently established for the user.

- $t G$-corresponds to the total number of goals defined in the coaching plan currently established for the user.

The following intervals relate to each emotional state based on the emotionalState value currently associated with the user progress:

- $\quad$ Sad-(0-1).

- Angry-(1-2).

- $\mathrm{OK}-(2-3)$.

- Happy-(3-4).

- $\quad$ Excited-(4-5).

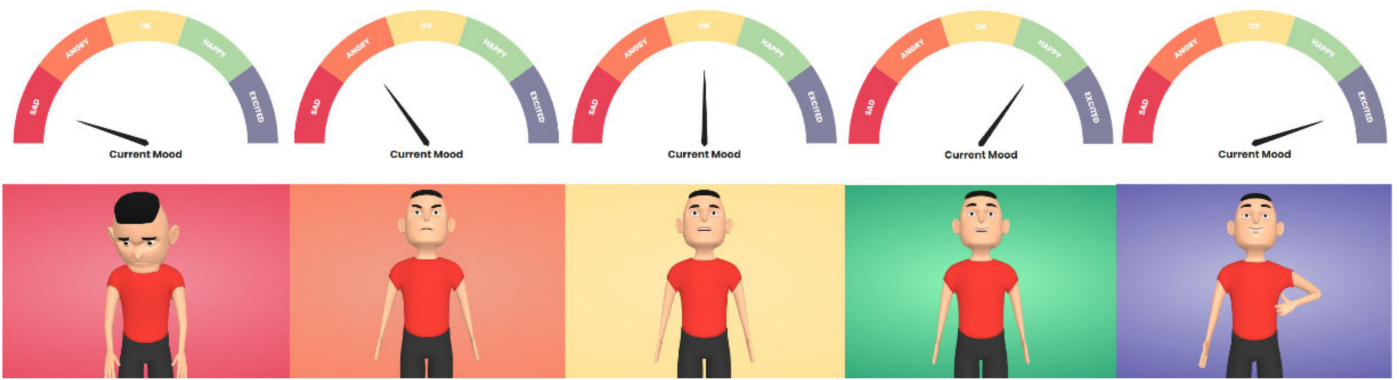

Figure 4. Representation of the five different emotional states of the cognitive assistant.

The cognitive assistant interacts with the user and exchanges different congratulatory or persuasive messages throughout the day explaining why he/she should make an effort to achieve an upcoming goal and why this is important to improve his/her current health condition (see Figure 5). 


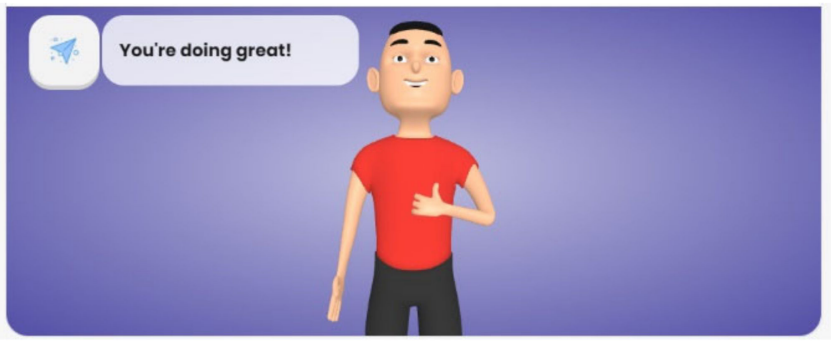

Figure 5. Representation of a message exchanged by the cognitive assistant.

Additionally, the user can access a detailed section, containing all the information on the progress of the coaching plans and showing relevant statistics related to the performance of the specific plan that was assigned to that user (see Figure 6).

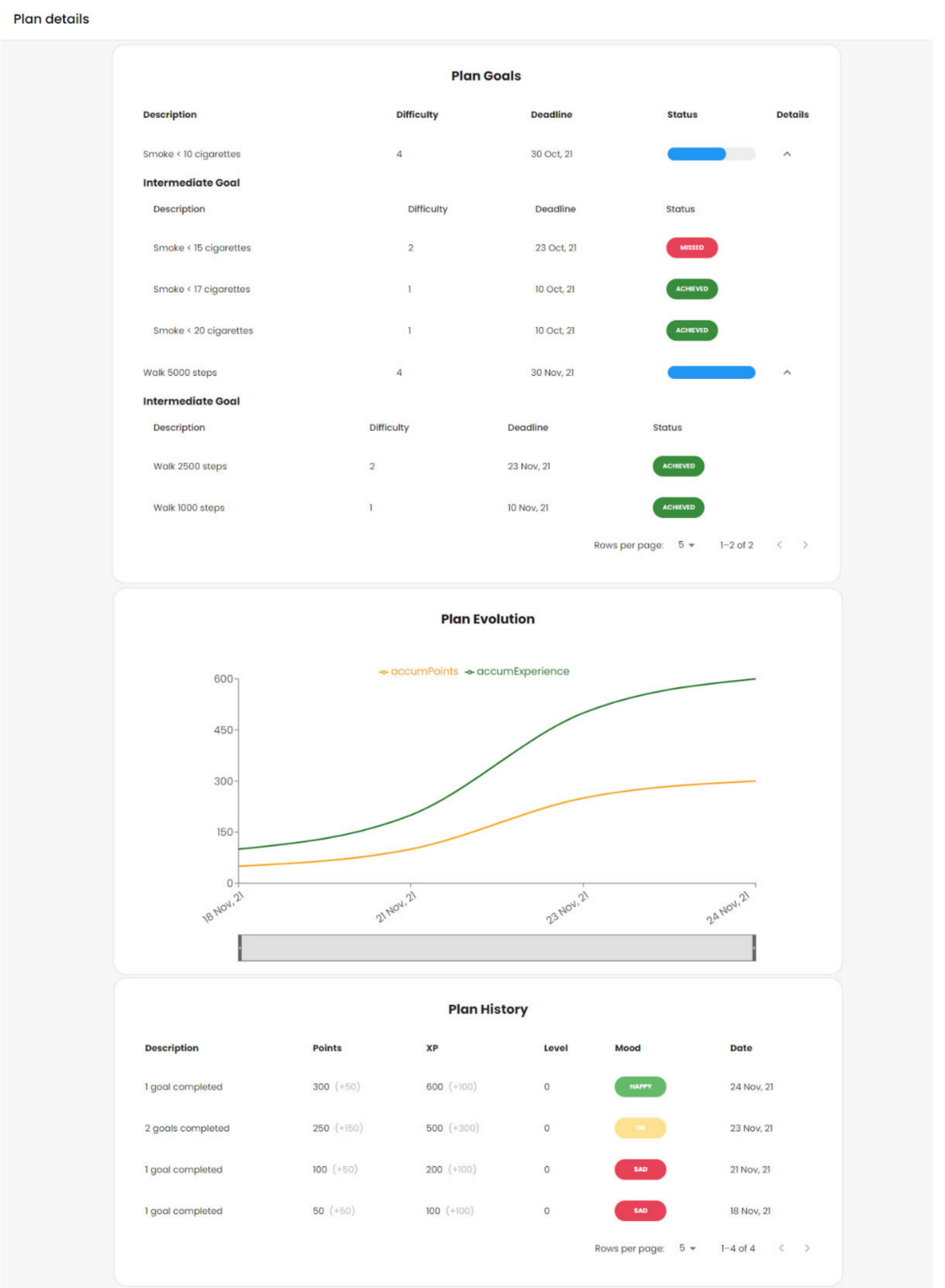

Figure 6. Coaching plan details interface. 
The coaching plan details include information on: (1) the status of each defined goal (whether the goal has already been achieved or not) and information on the intermediate goals needed to achieve a goal (for example, in Figure 6, different goals related to smoking habits are shown with intermediate goals for the user to smoke fewer than a certain number of cigarettes per day); (2) plan evaluation with the experience and points that are accumulated as the user achieves more goals; (3) plan history in terms of all the outcomes of the plan's execution (goals achieved, points obtained, experience gained, increase (or not) in the user level, and change in emotional state).

The coaching application also considers a marketplace in which the user can unlock additional content, as he is rewarded by successfully achieving positive health results. Currently, this additional content is strictly related to the customization of the cognitive assistant (see Figure 7), but other rewards could also be considered in the future (for example, content provided by family members of the elderly person such as congratulatory photos or videos).

Finally, the coaching application also includes a mini-games section (see Figure 8) in which the elderly person can participate in different cognitive challenges to not only improve his/her cognitive condition, but also to earn rewards. We have designed a tic-tactoe application with different difficulty levels. In the easier level, the user must defeat the computer and a random selection strategy is applied. In the harder level, the user must draw against the computer and a strategy using the minimax algorithm [30] is applied (computer never loses). The main reason for developing this section of the application was to define additional ways for the user to acquire points that can be exchanged for rewards, so that the user does not have to rely completely on the progress of any active coaching plans but instead can improve other aspects of his/her current health condition. This way, even if the user is struggling to achieve a certain goal (for example, the goal of improving physical conditions, such as walking a number of steps per day), he/she would still be able to make use of the application and acquire points, and consequentially increase their level, thus improving their overall player status.

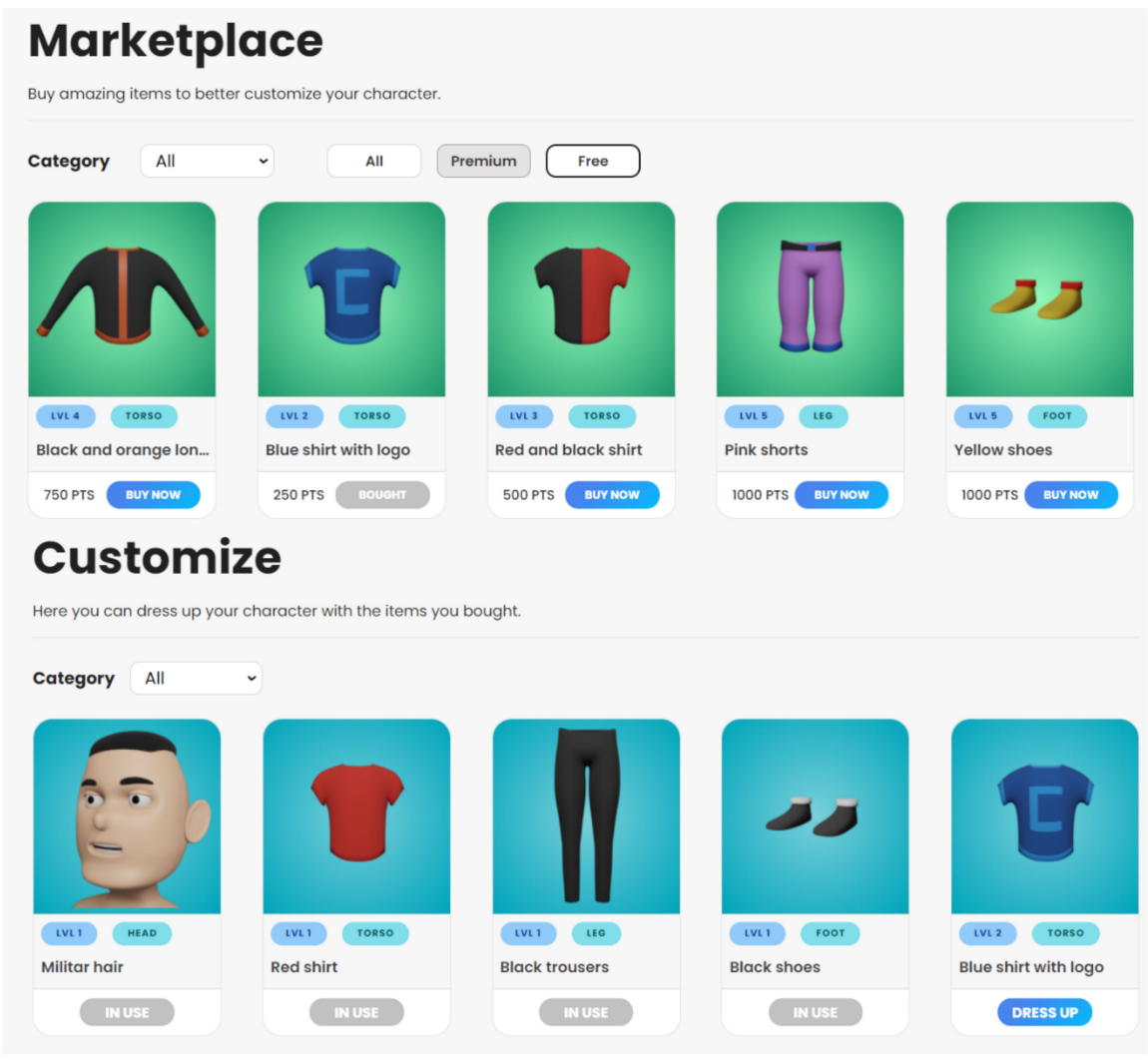

Figure 7. Cognitive assistant marketplace and customization interfaces. 


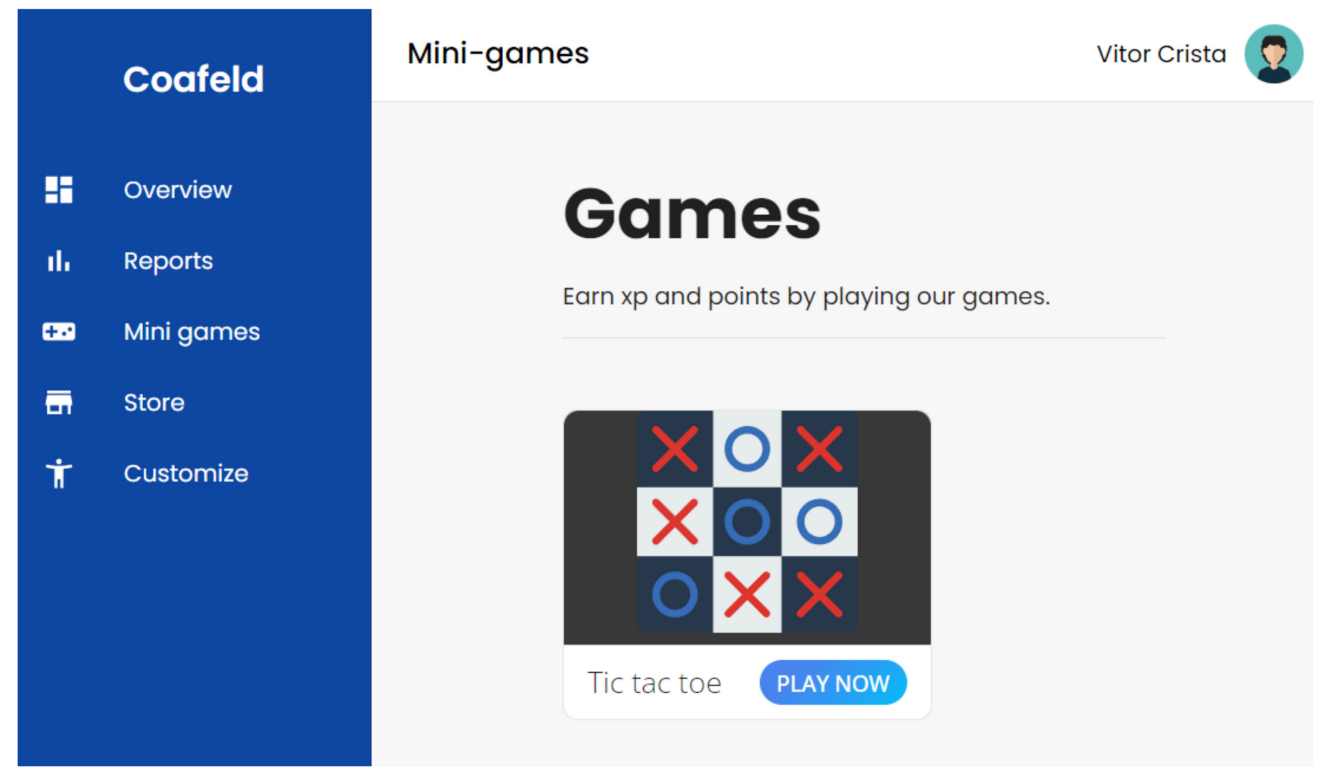

Figure 8. Cognitive games interface.

\subsection{API Gateway}

An API Gateway was developed in the proposed work with the main goal of encapsulating a set of several microservices and providing an API that is specific to each client (thus serving as a single-entry point into the system). It is also the responsibility of the API Gateway to include authorization/authentication functionalities, as well as assure asynchrnous communication between the requests sent to the available microservices. Three microservices were developed to store and provide information related to both user progress and health outputs.

- User Progress Microservice-This microservice was developed to manage the data related to user progress and actions within the coaching application. This includes all the information on current game experience/level/points; content unlocked; ingame purchases; cognitive play session results (games played, points and experience acquired, etc.); and virtual assistant configurations/emotional state.

- Coaching Microservice-This microservice was developed in a previous study [27], in which a generic mechanism was established to configure and evaluate coaching plans with health-related goals. These goals follow a certain health topic (for example, physical activity or smoking habits) and measure an input variable proided by the user as lower than/higher than/equal to a certain value or range. Another important aspect of these goals is the fact they can be dynamically updated with increasing/decreasing difficulty, depending on the performance of the user and whether he/she completed (or not) similar goals that were established for a certain coaching plan (following the example of smoking habits, a daily goal of smoking fewer than a certain number of cigarettes per day could be updated for the following days depending on whether the person achieved this goal or not) Additionally, this microservice provides information on user performance for each associated coaching plan (number of goals already achieved, number of goals failed, upcoming goals, goals' difficulty, etc.).

- Agent Microservice-This microservice was developed in a previous study [29] and comprised a Multi-Agent System developed in Jade [31] with the definition of two main agents that communicate with each other: A Personal Agent that returns the interaction (message queue), to be sent directly to the user, and evaluates the feedback provided by the user in terms whether the user achieved a health goal after performing a certain interaction with him/her; A Coaching Agent that applies a Reinforcement Learning strategy (using a Q-Learning algorithm) to understand which interactions should next be sent to the user at specific moments of the day based on the infor- 
mation exchanged with the Personal Agent and the interactions with the user. This Reinforcement Learing strategy allows the system to learn from the older person as he/she provides more feedback and understands which messages are most influential to motivate the person to achieve their health goals. Then, future interactions can be adapted according to this information.

\section{Results and Discussion}

After developing the coaching application, it was necessary to verify, from a technical perspective, if the system could correctly function and support the progress of the elderly person as a player and user of the system and provide him/her with feedback and motivational features depending on his/her performance and evolution. The coaching application was validated in three different simulation scenarios with three different levels of complexity. These scenarios have the same configurations as in [29]: in the first scenario, the cognitive assistant would exchange two different types of persuasive message; in the second scenario, the cognitive assistant would exchange four different persuasive messages; in the third scenario, the cognitive assistant would exchange six different persuasive messages.

For each scenario, a coaching plan was defined, with 30 daily health-related goals, which correspond to a time length of 30 days, and we studied both emotionalState and game point metrics, obtained using the coaching application and the completion of each established goal. The correct completion of an established goal rewards the user with (+100 Points), and the emotionalState was verified daily using the formula presented in the previous section of this work. Furthermore, 10 simulations were performed for each scenario and the results were compared in terms of the average values obtained.

To validate the coaching application, we compared the proposed approach using the Multi-Agent system developed in [29], considering two types of interactions with the user. One interaction would rely on the use of a Reinforcement Learning (RL) algorithm to identify the most effective persuasive message to send to the user at a certain moment of the day, while the other was a random strategy with the exchange of random persuasive messages (RANDOM).

For all the simulated scenarios, we considered the maximum number of interactions between the cognitive assistant and the user to be three messages per day, and that a goal would be achieved only after the user received the three most persuasive messages (according to his/her preferences). We chose this number specifically, due to the evidence in the literature [25] that recommends the exchange of messages at certain periods of the day, such as morning, lunch, and early evening, and these interactions would represent those moments of the day. Furthermore, the cognitive assistant could also exchange repeated types of messages during a day.

Next, the results obtained in each scenario are shown and discussed.

Looking at the results after 30 days of simulation, the first scenario (see Figure 9) obtained the best results among all three scenarios for both RL and RANDOM approaches in terms of the average number of points obtained and the emotional state of the cognitive assistant. In terms of values, the RL approach obtained an average of 2830 points and an emotional state of 4.72 (which corresponds to the excited state). On the other hand, the RANDOM approach obtained an average of 390 points and an emotional state of 0.65 (which corresponds to the sad state).

The second scenario (see Figure 10) obtained the second-best results among the three scenarios. In terms of values, the RL approach obtained an average of 2600 points and an emotional state of 4.33 (which corresponds to the excited state). On the other hand, the RANDOM approach obtained an average of 20 points and an emotional state of 0.03 (which corresponds to the sad state). 


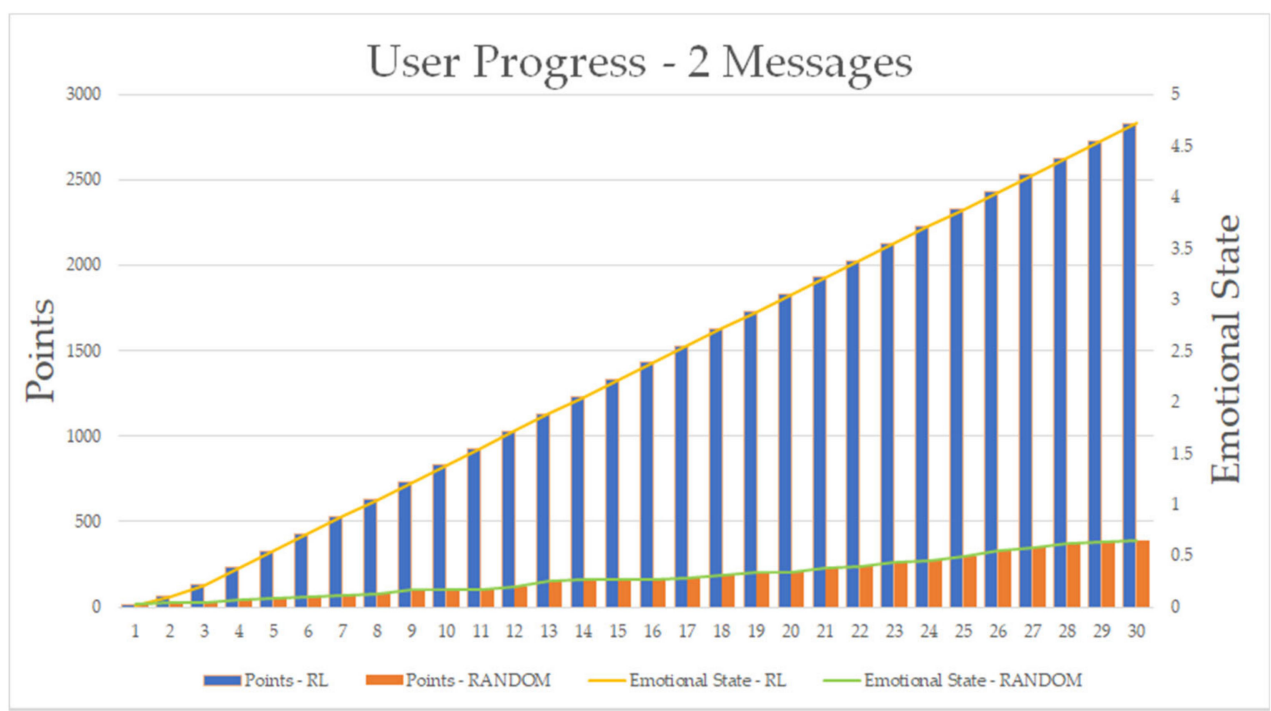

Figure 9. Simulation Results-1st Scenario.

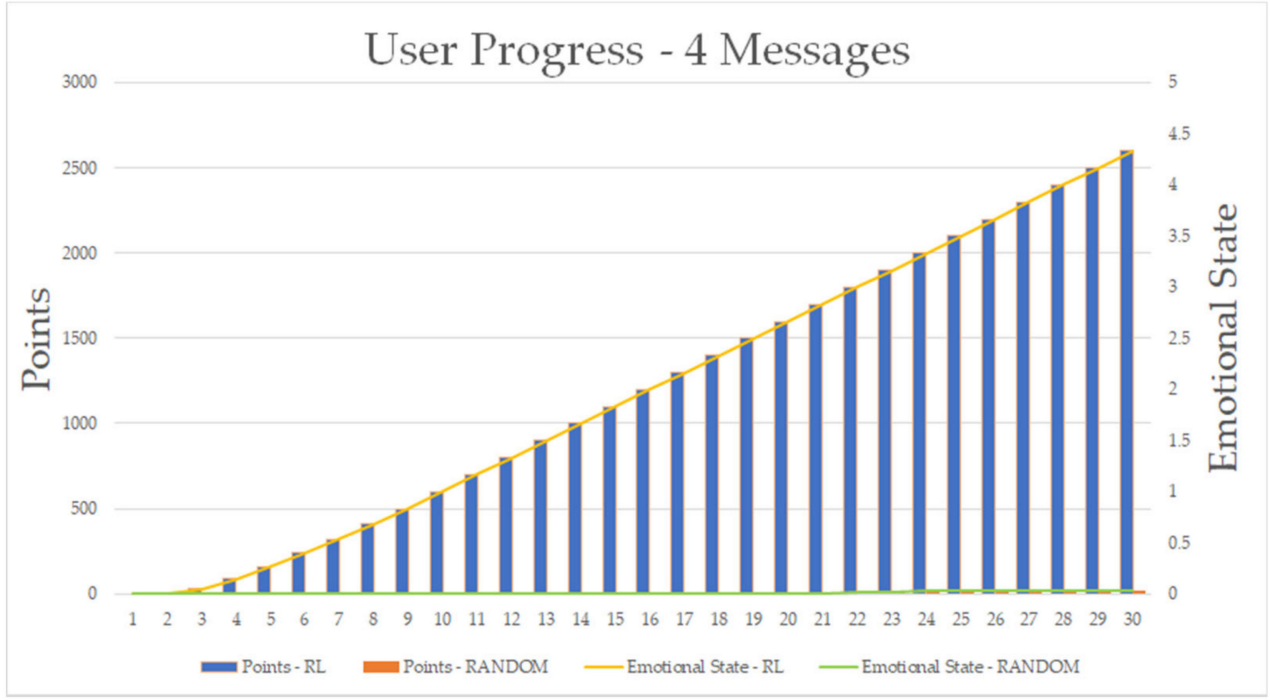

Figure 10. Simulation Results-2nd Scenario.

The third scenario (see Figure 11) obtained the worst results among the three scenarios. In terms of values, the RL approach obtained an average of 2380 points and an emotional state of 3.96 (which corresponds to the happy state). On the other hand, the RANDOM approach obtained an average of 20 points and an emotional state of 0.03 (which corresponds to the sad state).

Although it is beyond the scope of this work to prove which approach is superior (that study was conducted in [29], where we observed a clear outperformance of the RL approach compared to the RANDOM approach), it still important to highlight the very positive results of RL compared to RANDOM. In all three scenarios, it was possible to observe that the RL approach obtained very high results in terms of points and emotional state, even with higher complexity configurations. On the other hand, although the first scenario allowed the RANDOM approach to obtain a fair value in terms of points, in all scenarios, this approach obtained very low results. In terms of performance, we observed that our system could provide appropriate feedback to the user depending on his progress as a player of the application and the system could also provide adequate rewards as the user achieved more goals, by increasing the emotional state correctly and rewarding more 
points. At the same time, if the user struggled to achieve goals (which mostly correspond to the RANDOM approach) the system would not improve emotional state or reward points to the user. Still, it should be noted that the user was always capable of obtaining some points (at least 20 in the worst-case scenarios for the RANDOM case) and improve the emotional state of the cognitive assistant (the lowest value was 0.03 , which is still higher than the 0 baseline). Therefore, the system could recognize the user progress even in the worst-case scenarios (we could resemble those scenarios with the type of users that struggle the most to either improve their health condition or be motivated to do so) and reward the user accordingly. As such, we can conclude that the considered approach and the defined algorithm to represent the emotional state of the cognitive agent are adequate, and can confirm that it is possible to support the evolution of the elderly person as a player and user of the coaching system, which was the main goal of this study.

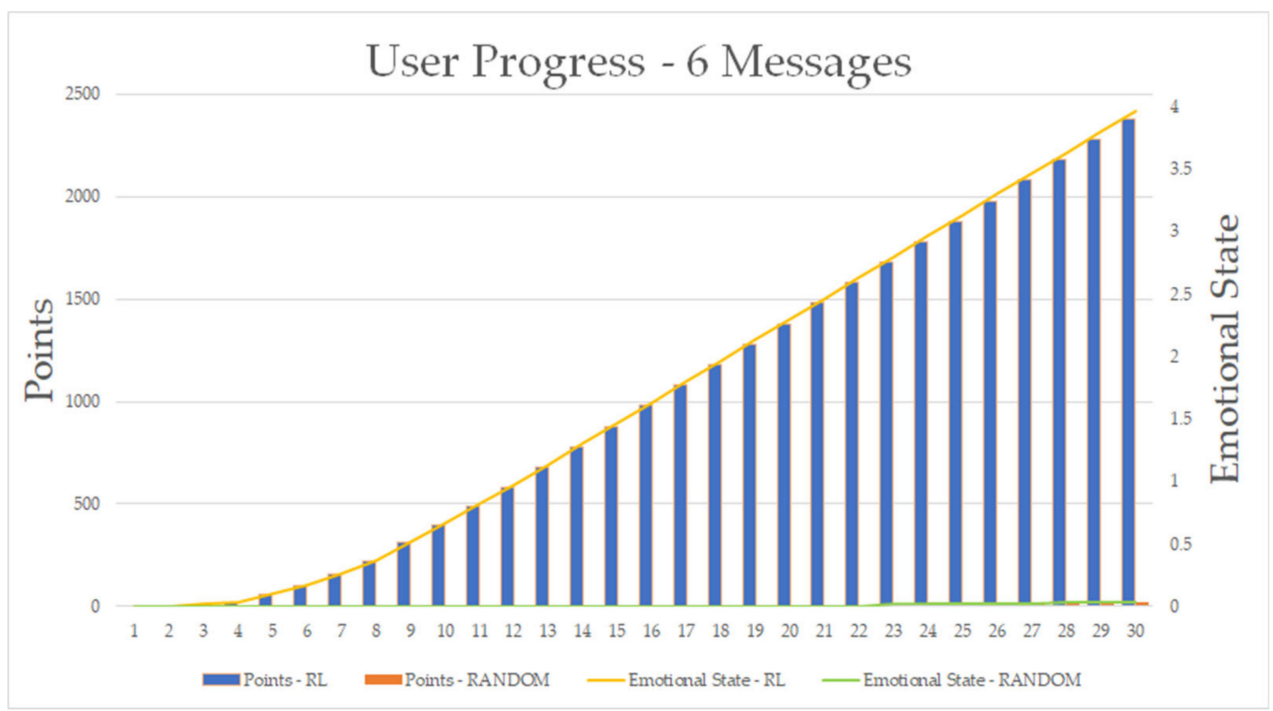

Figure 11. Simulation Results-3rd Scenario.

\section{Conclusions and Future Work}

With the increase in the elderly age group in the world's population, it is necessary to develop personalized healthcare systems capable of supporting older people in their daily living and promoting active and healthy aging. At the same time, attention has also been drawn to the definition of intelligent engagement strategies to persuade and motivate elderly people towards positive behavior changes. In this work, we follow propose a coaching system to support elderly people and offer different functionalities to motivate and persuade them to adopt a healthier lifestyle. We present a gamified coaching application with different elements to motivate the user and enhance user experience, and present a cognitive assistant to directly interact with the elderly person and provide him/her with feedback related to the progress of the user's health condition. We define different emotional states to represent the user progress, which will be positive as the user's health condition is improved or increasingly negative as the user's health condition is decreased. Finally, we validate our coaching system in terms of the support and feedback provided to the user according to his progress and interactions with the cognitive assistant.

As future work, we intend to improve the proposed coaching system to consider higher-complexity scenarios and people with more complex behaviors and ways of living. Additionally, we intend to incorporate our approach with devices that can provide realtime data and directly monitor the health condition of the patient, such as smartbands to measure the accuracy and impact of each interaction between the proposed system and the elderly person. We also intend to improve our gamified system by: (1) including additional users (family) that can provide additional content and rewards to the elderly person; (2) establishing cognitive plans that can be evaluated based on the results drawn 
from the cognitive games section of the application. Finally, we are aware of the necessity of validating the developed system within the elderly population to obtain more results and conclusions in terms of usability and acceptability.

With these points in mind, it will be possible to present an intelligent approach that correctly supports the elderly person during his/her daily life and promotes and motivates active and healthy ageing.

Author Contributions: Conceptualization, D.M. and J.C.; methodology, D.M. and J.C.; software, D.M. and V.C.; validation, D.M. and V.C.; formal analysis, D.M.; investigation, D.M.; resources, G.M.; data curation, D.M. and V.C.; writing-original draft preparation, D.M.; writing-review and editing, D.M., V.C., J.C., J.M.C. and G.M.; visualization, D.M.; supervision, J.C., J.M.C. and G.M.; project administration, G.M.; funding acquisition, G.M. All authors have read and agreed to the published version of the manuscript.

Funding: The work presented in this paper has been developed under the EUREKA-ITEA3 Project PHE (PHE-16040), and by National Funds through FCT (Fundação para a Ciência e a Tecnologia) under the under the project UIDP/00760/2020 and by NORTE-01-0247-FEDER-033275 (AIRDOC-“"Aplicação móvel Inteligente para suporte individualizado e monitorização da função e sons Respiratórios de Doentes Obstrutivos Crónicos ") by NORTE 2020 (Programa Operacional Regional do Norte).

Data Availability Statement: Experimental data is available upon request.

Conflicts of Interest: The authors declare no conflict of interest.

\section{References}

1. United Nations. World Population Ageing Report; United Nations: San Francisco, CA, USA, 2019.

2. World Health Organization. Ageing; World Health Organization: Geneva, Switzerland, 2020.

3. Martinho, D.; Carneiro, J.; Corchado, J.M.; Marreiros, G. A systematic review of gamification techniques applied to elderly care. Artif. Intell. Rev. 2020, 53, 4863-4901. [CrossRef]

4. Hermens, H.; op den Akker, H.; Tabak, M.; Wijsman, J.; Vollenbroek, M. Personalized Coaching Systems to support healthy behavior in people with chronic conditions. J. Electromyogr. Kinesiol. 2014, 24, 815-826. [CrossRef] [PubMed]

5. Degeling, K.; Koffijberg, H.; Ijzerman, M.J. A systematic review and checklist presenting the main challenges for health economic modeling in personalized medicine: Towards implementing patient-level models. Expert Rev. Pharm. Outcomes Res. 2017, 17, 17-25. [CrossRef] [PubMed]

6. Thakur, N.; Han, C.Y. Framework for a personalized intelligent assistant to elderly people for activities of daily living. Int. J. Recent Trends Hum. Comput. Interact. 2019, 9, 1-22.

7. Costa, A.; Heras, S.; Palanca, J.; Jordán, J.; Novais, P.; Julián, V. Argumentation schemes for events suggestion in an e-Health platform. In Proceedings of the International Conference on Persuasive Technology, Amsterdam, The Netherlands, 4-6 April 2017; Springer: Berlin/Heidelberg, Germany, 2017; pp. 17-30.

8. Costa, A.; Heras, S.; Palanca, J.; Novais, P.; Julián, V. A persuasive cognitive assistant system. In Proceedings of the International Symposium on Ambient Intelligence, Sevilla, Spain, 1-3 June 2016; Springer: Berlin/Heidelberg, Germany, 2016; pp. 151-160.

9. Costa, A.; Heras, S.; Palanca, J.; Novais, P.; Julián, V. Persuasion and Recommendation System Applied to a Cognitive Assistant. Adv. Distrib. Comput. Artif. Intell. J. 2016, 5, 89-99. [CrossRef]

10. Stokes, B. Videogames have changed: Time to considerSerious Games'? Dev. Educ. J. 2005, 11, 12.

11. Whitehead, A.; Johnston, H.; Nixon, N.; Welch, J. Exergame effectiveness: What the numbers can tell us. In Proceedings of the 5th ACM SIGGRAPH Symposium on Video Games, Los Angeles CA, USA, 28-29 July 2010; ACM: New York, NY, USA, 2010; pp. 55-62.

12. Hamari, J.; Koivisto, J.; Sarsa, H. Does gamification work?-A literature review of empirical studies on gamification. In Proceedings of the 2014 47th Hawaii International Conference on System Sciences (HICSS), Waikoloa, HI, USA, 6-9 January 2014; IEEE: Piscataway, NJ, USA, 2014; pp. 3025-3034.

13. Schultz, T.; Putze, F.; Steinert, L.; Mikut, R.; Depner, A.; Kruse, A.; Franz, I.; Gaerte, P.; Dimitrov, T.; Gehrig, T. I-CARE-An Interaction System for the Individual Activation of People with Dementia. Geriatrics 2021, 6, 51. [CrossRef] [PubMed]

14. Puig, J.; Echeverría, P.; Lluch, T.; Herms, J.; Estany, C.; Bonjoch, A.; Ornelas, A.; París, D.; Loste, C.; Sarquella, M. A Specific Mobile Health Application for Older HIV-Infected Patients: Usability and Patient's Satisfaction. Telemed. e-Health 2021, 27, $432-440$. [CrossRef] [PubMed]

15. Gellner, C.; Buchem, I.; Müller, J. Application of the Octalysis Framework to Gamification Designs for the Elderly. In Proceedings of the European Conference on Games Based Learning, Brighton, UK, 23-24 September 2021; Academic Conferences International Limited: London, UK, 2021; p. 260-XIX.

16. Kostopoulos, P.; Kyritsis, A.I.; Ricard, V.; Deriaz, M.; Konstantas, D. Enhance daily live and health of elderly people. Procedia Comput. Sci. 2018, 130, 967-972. [CrossRef] 
17. Balsa, J.; Félix, I.; Cláudio, A.P.; Carmo, M.B.; Silva, I.C.; Guerreiro, A.; Guedes, M.; Henriques, A.; Guerreiro, M.P. Usability of an Intelligent Virtual Assistant for Promoting Behavior Change and Self-Care in Older People with Type 2 Diabetes. J. Med. Syst. 2020, 44, 130. [CrossRef] [PubMed]

18. Chan, J.; Nejat, G. Promoting engagement in cognitively stimulating activities using an intelligent socially assistive robot. In Proceedings of the 2010 IEEE/ASME International Conference on Advanced Intelligent Mechatronics, Montreal, QC, Canada, 6-9 July 2020; pp. 533-538.

19. Costa, A.; Novais, P.; Julian, V.A. A survey of cognitive assistants. In Personal Assistants: Emerging Computational Technologies; Springer: Berlin/Heidelberg, Germany, 2018; pp. 3-16.

20. De Barcelos Silva, A.; Gomes, M.M.; da Costa, C.A.; da Rosa Righi, R.; Barbosa, J.L.V.; Pessin, G.; De Doncker, G.; Federizzi, G. Intelligent personal assistants: A systematic literature review. Expert Syst. Appl. 2020, 147, 113193. [CrossRef]

21. Ramos, J.; Oliveira, T.; Satoh, K.; Neves, J.; Novais, P. Cognitive assistants-An analysis and future trends based on speculative default reasoning. Appl. Sci. 2018, 8, 742. [CrossRef]

22. Le, N.-T.; Wartschinski, L. A Cognitive Assistant for improving human reasoning skills. Int. J. Hum.-Comput. Stud. 2018, 117, 45-54. [CrossRef]

23. Kolenik, T.; Gams, M. Intelligent Cognitive Assistants for Attitude and Behavior Change Support in Mental Health: State-of-theArt Technical Review. Electronics 2021, 10, 1250. [CrossRef]

24. Chang, E. An Exploration of Virtual Pets and Our Relationships to Them. Ph.D. Thesis, University of California Santa Luz, San Diego, CA, USA, 2020.

25. Ferwerda, B.; Lee, M. Tamagotchi++: A Serious, Personalized Game to Encourage Healthy Behavior. In Proceedings of the 24th International on Intelligent User Interfaces (IUI), CEUR-WS. Los Angeles, CA, USA, 16-20 March 2019.

26. Cheok, A.D.; Zhang, E.Y. Emotional Relationships with Robotic Companions. In Human-Robot Intimate Relationships; Springer: Berlin/Heidelberg, Germany, 2019; pp. 153-158.

27. Martinho, D.; Vieira, A.; Carneiro, J.; Martins, C.; Almeida, A.; Marreiros, G. A Definition of a Coaching Plan to Guide Patients with Chronic Obstructive Respiratory Diseases. In Proceedings of the World Conference on Information Systems and Technologies, Budva, Montenegro, 7-10 April 2020; Springer: Berlin/Heidelberg, Germany, 2020; pp. 54-64.

28. Martinho, D.; Carneiro, J.; Novais, P.; Neves, J.; Corchado, J.; Marreiros, G. A conceptual approach to enhance the well-being of elderly people. In Proceedings of the EPIA Conference on Artificial Intelligence, Vila Real, Spain, 3-6 September 2019; Springer: Berlin/Heidelberg, Germany, 2019; pp. 50-61.

29. Martinho, D.; Carneiro, J.; Neves, J.; Novais, P.; Corchado, J.; Marreiros, G. A Reinforcement Learning Approach to Improve User Achievement of Health-Related Goals. In Proceedings of the EPIA Conference on Artificial Intelligence, Virtual Event, 7-9 September 2021; Springer: Berlin/Heidelberg, Germany, 2021; pp. 266-277.

30. Savelli, R.M.; de Beauclair Seixas, R. Tic-Tac-Toe and the Minimax Decision Algorithm. Available online: https://www.lua.org/gems/ (accessed on 28 December 2021).

31. Bellifemine, F.; Poggi, A.; Rimassa, G. Developing multi-agent systems with JADE. In Proceedings of the International Workshop on Agent Theories, Architectures, and Languages, Montreal, QC, Canada, 19-20 August 1995; Springer: Berlin/Heidelberg, Germany, 2000; pp. 89-103. 